\title{
Studies of the Organ Distribution in Mice of Teniposide Liposomes Designed for Treatment of Diseases in the Mononuclear Phagocytic System
}

\author{
EVA LILIEMARK, JAN LILIEMARK, NILS KÅLLBERG, MAGNUS BJÖRKHOLM, BRITA \\ SJÖSTRÖM, AND CURT PETERSON \\ Departments of Clinical Pharmacology [E.L, J.L., C.P.] and Medicine, Section of Hematology and \\ Medical Immunology [M.B.], and Karolinska Pharmacy [N.K.], Karolinska Hospital, Stockholm, and \\ Institute for Surface Chemistry [B.S.], Stockholm, Sweden
}

\section{ABSTRACT}

Liposomes can be used for the delivery of drugs in cancer chemotherapy. After i.v. injection liposomes are to a large extent taken up by the mononuclear phagocytic system (MPS). When treating diseases in the MPS, such as the histiocytic syndromes, this property is of potential value for drug targeting and may lead to a more efficient therapy with less systemic toxicity. Teniposide (VM-26) is a potent anti-tumor drug. Its lipophilicity makes it suitable for liposomal formulation. Teniposide liposomes were prepared by dissolving egg phosphatidylcholine and dioleoyl phosphatidic acid (19:1 molar ratio) in methylene chloride together with teniposide. After solvent evaporation, the dry lipid film was dispersed in a glucose solution $(50 \mathrm{mg} / \mathrm{mL})$, and size calibration was obtained by filtration through polycarbonate filters. The amount of teniposide incorporated was $2.5 \mathrm{~mol} \%$. To investigate the organ distribution, teniposide liposomes contain- ing radiolabeled teniposide or phospholipid were given i.v. to mice. By increasing the size of the vesicles, the MPS uptake could be modulated. When vesicles of $200 \mathrm{~nm}$ and 1 and $3 \mu \mathrm{m}$ were injected, the drug levels in the spleen were increased 2.6-, 6.8-, and 21-fold $40 \mathrm{~min}$ after injection, compared with levels after injection of the commercial teniposide formulation. It was concluded that organ distribution of teniposide in mice could be modified by administering the drug in liposomal form with the potential of improved treatment of diseases engaging the MPS. (Pediatr Res 38: 7-10, 1995)

\section{Abbreviations}

FHL, familial hemophagocytic lymphohistiocytosis

MPS, mononuclear phagocytic system

PC, phosphatidyl choline
Most anti-tumor agents have severe side effects limiting the therapeutic possibilities when given systemically. Selective localization of anti-tumor drugs may improve treatment results of chemotherapy. After i.v. injection most liposomes are rapidly cleared from the circulation by phagocytosis by the MPS in liver, spleen, and bone marrow (1). Liposome-mediated drug delivery is therefore an alternative if the target is tissues of the MPS.

The podophyllotoxin derivative teniposide is active against solid tumors such as small cell lung cancer and germinal tumors as well as hematologic malignancies $(2,3)$, and lipidsoluble drugs such as teniposide can easily be encapsulated within the membrane of liposomes (4).

The podophyllotoxins are the drugs of choice in the treatment of FHL, a rare childhood disease which affects the macrophages in liver and spleen $(5,6)$. Bone marrow toxicity

Received May 26, 1994; accepted January 26, 1995.

Correspondence and reprint requests: Eva Liliemark, Department of Clinical Pharmacology, Karolinska Hospital, S-171 76 Stockholm, Sweden.

Supported by grants from the Swedish Cancer Society, the Swedish Children Cancer Society, and Bristol Myers-Squibb in Sweden. is dose-limiting, and liposomal formulations could improve the therapeutic index of teniposide. Liposomal teniposide therapy might also be of value for other malignant and autoimmune disorders involving phagocytic cells.

The purpose of this work was to prepare and evaluate teniposide liposomes in an attempt to achieve enhanced drug uptake in the MPS. Mice were used for drug distribution studies.

\section{METHODS}

Chemicals. $\left[{ }^{14} \mathrm{C}\right]$ Teniposide, specific activity $0.77 \mathrm{GBq} /$ mmol, was kindly provided by Dr Kathleen Deardorff, Bristol Myers-Squibb (Syracuse, NY). Teniposide in the commercially available formulation, Vumon, was received as a gift from Bristol-Myers Squibb (Bromma, Sweden).

Phosphatidylcholine, $\mathfrak{l}-\alpha$-[2-palmitoyl-1- $\left.{ }^{14} \mathrm{C}\right]$ dipalmitoyl, specific activity $2.12 \mathrm{GBq} / \mathrm{mmol}$, radiochemical purity $99 \%$, and phosphatidylcholine, L- $\alpha$-[choline-methyl- $\left.{ }^{3} \mathrm{H}\right]$ dipalmitoyl, specific activity $1480 \mathrm{GBq} / \mathrm{mmol}$, radiochemical purity $99 \%$ was purchased from NEN Research Products, Du Pont Scan- 
dinavia A.B. (Stockholm, Sweden). Phosphatidylcholine, L- $\alpha$ [2-palmitoyl-1- ${ }^{14} \mathrm{C}$ ]dipalmitoyl with specific activity $4.2 \mathrm{GBq} /$ mmol, radiochemical purity 99\%, from Amersham International (Amersham, UK) was also used. Egg PC and dioleoyl phosphatidic acid were purchased from Avanti Polar Lipids, Inc. (Pelham, Ala). All solvents used were of analytical or HPLC grade.

Preparation and characterization of liposomes. Liposomes were produced in small batches the day before use in animal experiments. The phospholipids, PC, $138 \mathrm{mg}$, and phosphatidic acid, $6.2 \mathrm{mg}$ (19:1 molar ratio), were dissolved in $10 \mathrm{~mL}$ methylene chloride together with teniposide, $2.9 \mathrm{mg}$. The radiolabeled teniposide or $\mathrm{PC}$ was added to the solvent. After evaporation, the dry lipid film on the inside walls of the flask was dispersed in $8.8 \mathrm{~mL}$ glucose solution $(50 \mathrm{mg} / \mathrm{mL})$ by manual shaking. Large unilamellar vesicles were produced by repeated filtration through Nucleopore polycarbonate filters, Costar Corp. (Cambridge, MA), with different pore sizes (200 nm and 1 and $3 \mu \mathrm{m}$ ) (7). The Extruder Lipex Biomembranes Inc. (Vancouver, Canada), was used to produce vesicles by extrusion two to three times through double 200-nm filters. Larger vesicles were filtered manually two to four times through membranes with $1-$ and $3-\mu \mathrm{m}$ pores. The teniposide concentration of the preparations was $365 \mu \mathrm{g} / \mathrm{mL}$, and the phospholipid concentration $16.4 \mathrm{mg} / \mathrm{mL}$.

Teniposide concentrations in the preparations were determined by reversed phase high performance liquid chromatography using a Spherisorb Phenomenex mini-bore column eluted with methanol/water/acetic acid (30:69:1). Detection was performed fluorometrically, $\lambda$ excitation/emission $=230$ / 330. The ${ }^{14} \mathrm{C}$ or ${ }^{3} \mathrm{H}$ radioactivity in the preparations was determined by $\beta$-counting using a Packard Tri-Carb $460 \mathrm{C}$ liquid scintillation system. The mean diameter of the 1 - and $3-\mu \mathrm{m}$ liposomes was measured by laser diffraction with a Mastersizer, Malvern Instruments (Malvern, UK), and the size of the $200-\mathrm{nm}$ liposomes was measured by dynamic light scattering at a fixed angle of $90^{\circ}$ using an Autosizer IIc, Malvern Instruments (Malvern, UK). The unilamellar structure of the vesicles has been verified with cryo-transmission electron microscopy as previously described (8).

Preparation of radiolabeled teniposide. Radiolabeled teniposide was obtained by dissolving $\left[{ }^{14} \mathrm{C}\right]$ teniposide in methanol and evaporating to dryness under nitrogen in a glass tube. The commercially available teniposide solution, Vumon, containing cremophore, was used to solubilize the radiolabeled teniposide. Vumon, $290 \mu \mathrm{L}$ (teniposide concentration $10 \mathrm{mg} / \mathrm{mL}$ ), was added, and the tube was vortexed for $5 \mathrm{~min}$. The mixture was diluted with glucose solution $(50 \mathrm{mg} / \mathrm{mL})$ to $8.8 \mathrm{~mL}$. The teniposide concentration in the solution was $365 \mu \mathrm{g} / \mathrm{mL}$. The phase equilibrium in the solubilization system was not expected to be affected by the very small addition of teniposide. However, to minimize the risk of precipitation of teniposide crystals, the solution of radiolabeled teniposide for administration of free drug was prepared immediately before use.

Animal study. The experiments were approved by the local ethics committee for animal studies and performed as previously described by Masquelier et al. (9). Female NMRI mice $(25-35 \mathrm{~g})$ were used for the experiments. After $5 \mathrm{~min}$ of warming of the animal at $45^{\circ} \mathrm{C}$ to obtain vasodilatation, $150-$ $200 \mu \mathrm{L}$ of liposomal or free teniposide were injected in a tail vein. At different time intervals, the mice were anesthetized by diethyl ether. Blood obtained by neck incision was collected in heparin tubes and kept on ice until plasma was separated by centrifugation. Organs were washed in ice-cooled PBS, pH 7.4, weighed, and homogenized. Radioactivity in plasma, liver, spleen, lung, and heart was determined by liquid scintillation counting.

One or two experiments using one or two animals per time point was performed with free teniposide and liposomes labeled with $\left[{ }^{14} \mathrm{C}\right]$ teniposide, liposomes labeled with $\left[{ }^{14} \mathrm{C}\right] \mathrm{PC}$, and liposomes labeled with both $\left[{ }^{14} \mathrm{C}\right]$ teniposide and $\left[{ }^{3} \mathrm{H}\right] \mathrm{PC}$. With double labeling the fate of both drug and lipid could be followed in the same animal. By dividing radioactivity in the samples with the injected amount of radioactivity per gram body weight of the mice, the results from the different experiments could be compared.

\section{RESULTS}

In the attempts to optimize the amount of teniposide possible to incorporate into our liposomes, drug/lipid molar ratios of $1-15 \%$ were studied. The recovery of teniposide was $>90 \%$ when the teniposide molar ratio was up to $7 \%$. Stability tests were performed on liposomes extruded twice through double 200-nm polycarbonate filters. Drug crystals occurred if the teniposide content was $3 \%$ or higher. With $2 \mathrm{~mol} \%$ teniposide the liposomes could be stored at $+8^{\circ} \mathrm{C}$ for 3 mo without formation of drug crystals as judged from the results of filtration through $1-\mu \mathrm{m}$ polycarbonate filters without significant loss of teniposide and from microscopic examination in polarized light. The mean liposome diameter was 200-300 nm and the size distribution was homogenous, reproducible, and stable. The liposomes were stable for at least 3 mo in PBS, pH 7.4, and in glucose, $50 \mathrm{mg} / \mathrm{mL}$.

During the filtration procedure for liposome production the size distribution approached the size of the pores with number of passages through the filter. Figure 1 illustrates how the number of passages affect mean liposome size using a 3- $\mu \mathrm{m}$ polycarbonate membrane. After four filtrations the size is rather homogenous with a mean diameter of $3.5 \mu \mathrm{m}$, and $95 \%$ of the liposomes are below $7 \mu \mathrm{m}$ (volume distribution), which means smaller than the red blood cells.

To evaluate the in vivo distribution of teniposide liposomes we used mice and injected the commercial formulation of teniposide, Vumon, as a reference. The liposome preparations injected were identical in phospholipid and drug content and labeled with either radioactive teniposide, radioactive phosphatidyl choline, or both. The teniposide content was $2.5 \mathrm{~mol}$ $\%$. The plasma clearances of free teniposide and the five various liposomal formulations are shown in Figure 2. The liposomal teniposide has a slightly delayed clearance compared with free drug, and a pronounced delay is observed of the lipid clearance. The initial distribution phase of free teniposide is extremely rapid with a half-life of $1-2 \mathrm{~min}$. A slower phase is seen between $5-10 \mathrm{~min}$ and $4 \mathrm{~h}$. The liposomal formulation is delaying the initial distribution of the drug. However, after the 


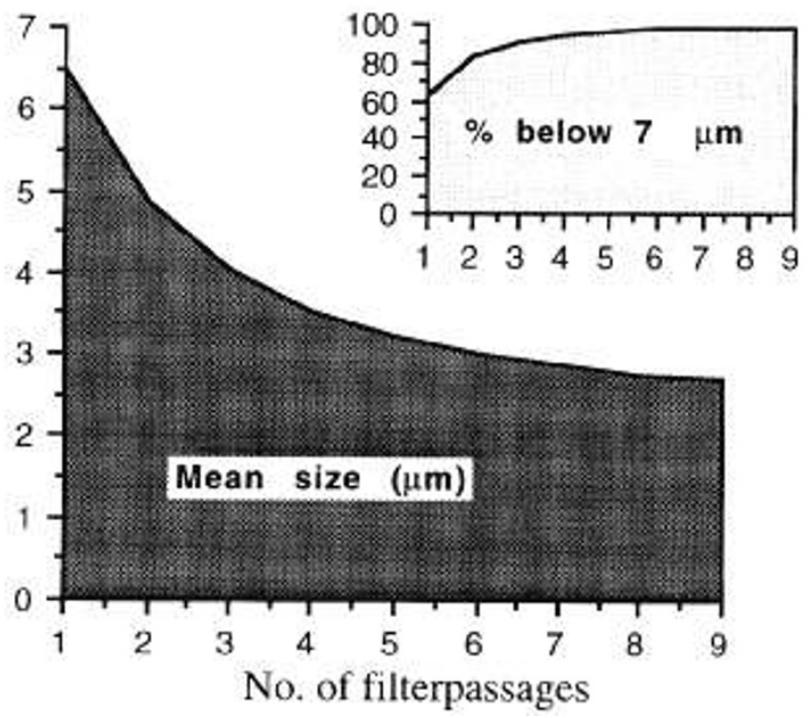

Figure 1. Filtration of teniposide liposomes through 3- $\mu \mathrm{m}$ polycarbonate filters. Mean size of the vesicles is plotted against number of filter passages. Inset illustrates percentage below $7 \mu \mathrm{m}$, the size of the red blood cells.

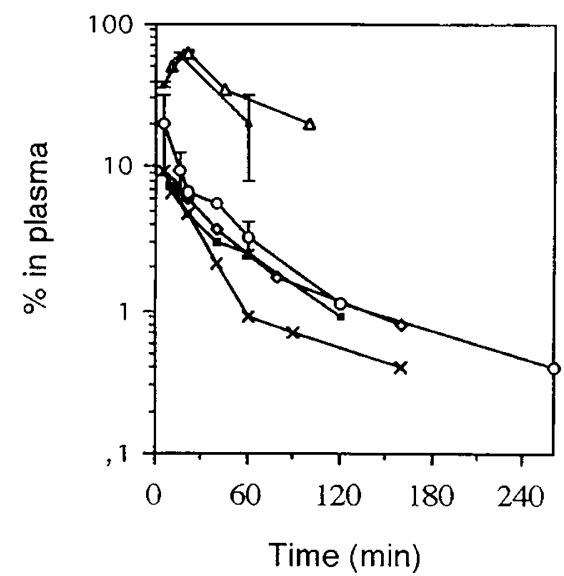

Figure 2. Plasma clearance of $\left[{ }^{14} \mathrm{C}\right]$ teniposide administered as free drug $(\times-X)$ or incorporated into liposomes of different sizes: $200 \mathrm{~nm}$ $(\diamond-\diamond), 1 \mu \mathrm{m}(\mathbf{\square})$, or $3 \mu \mathrm{m}(\mathrm{O}-\mathrm{O})$ and of liposomes labeled with $\left[{ }^{3} \mathrm{H}\right] \mathrm{PC}$ or $\left[{ }^{14} \mathrm{C}\right] \mathrm{PC}$ of $200 \mathrm{~nm}(\triangle-\triangle)$ or $3 \mu \mathrm{m}(\boldsymbol{\Lambda}-\boldsymbol{\Lambda})$. The remaining percentage of the injected radioactivity is calculated for each time point. The points represent one to three animals, and the bars show the standard deviation from the mean when more than one animal was used.

distribution phase the slope of the elimination curves of liposomal teniposide approaches that of the free drug. The terminal half-life cannot be accurately determined from these data.

The organ distribution of radiolabeled teniposide administered in liposomes was different from that of the free drug. The distribution to liver, spleen, lung, and heart $60 \mathrm{~min}$ post i.v. injection of free teniposide, 1- and 3- $\mu \mathrm{m}\left[{ }^{14} \mathrm{C}\right]$ teniposide liposomes is illustrated in Figure 3. Especially the drug levels in the spleen increased considerably. Liposomes of $200 \mathrm{~nm}$ and 1 and $3 \mu \mathrm{m}$ produced a $2.6-, 6.8-$, and 21 -fold increase in splenic concentrations of teniposide $40 \mathrm{~min}$ post injection compared with administration of free drug. Drug levels in the liver were generally high, and the accumulation in lung and heart was low.

Liposome size affects splenic uptake. Figure 4 shows the drug distribution in the spleen post injection of free teniposide

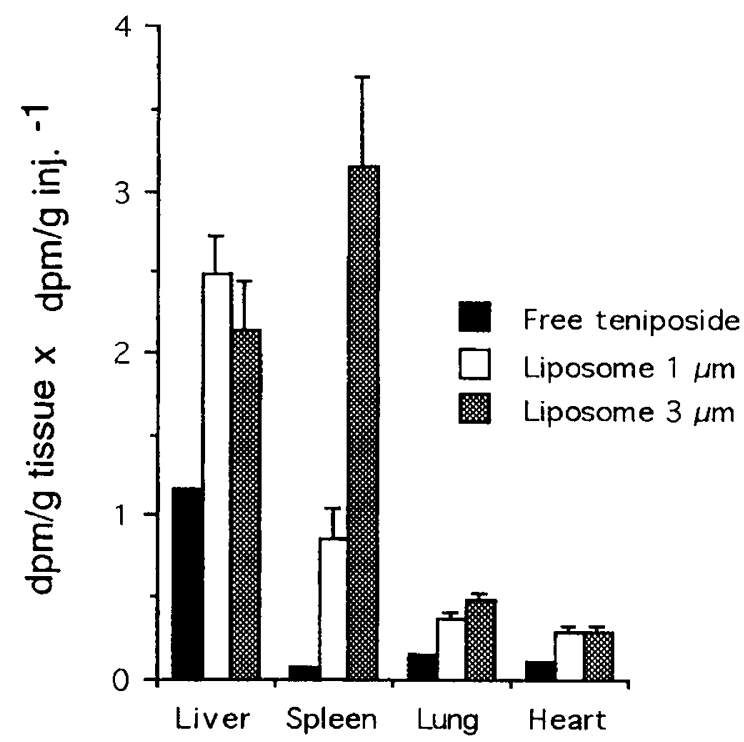

Figure 3. The distribution to blood, liver, spleen, lung, and the heart $60 \mathrm{~min}$ post i.v. injection of free teniposide, and 1- and 3- $\mu \mathrm{m}$ teniposide lipsosomes. Uptake is expressed as radioactivity per gram of tissue in the samples divided by injected amount per gram of body weight of the mice. One animal was used for free teniposide, two for 1- $\mu \mathrm{m}$ liposomes, and three for 3- $\mu \mathrm{m}$ liposomes. Bars show the standard deviation.

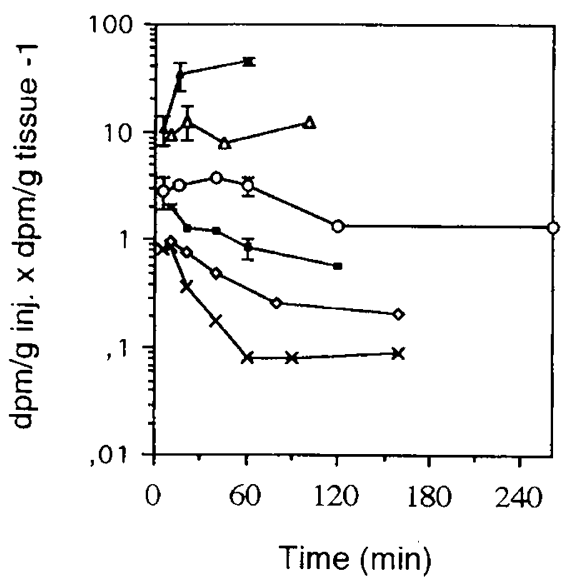

Figure 4. Accumulation in the spleen of $\left[{ }^{14} \mathrm{C}\right]$ teniposide administered as free drug $(\times-\times)$ or incorporated into liposomes of $200 \mathrm{~nm}(\diamond-\diamond), 1 \mu \mathrm{m}$ (1- or $3 \mu \mathrm{m}(\mathrm{O}-\mathrm{O})$ and of lipsomes labeled with $\left[{ }^{3} \mathrm{H}\right] \mathrm{PC}$ or $\left[{ }^{14} \mathrm{C}\right] \mathrm{PC} 200 \mathrm{~nm}(\triangle-\triangle)$ or $3 \mu \mathrm{m}(\boldsymbol{\Delta}-\mathbf{\Delta})$. Uptake is expressed as radioactivity (dpm) per gram of tissue in the samples divided by injected amount per gram of body weight of the mice. The points represent one to three animals and the bars show the standard deviation from the mean when more than one animal was used.

and liposomes of various sizes. When liposomes were labeled with $\left[{ }^{14} \mathrm{C}\right] \mathrm{PC}$ or $\left[{ }^{3} \mathrm{H}\right] \mathrm{PC}$ the distribution of phospholipid differed from that of the drug, suggesting in vivo leakage of drug or transfer of phospholipid from the liposomes.

\section{DISCUSSION}

Successful anti-cancer chemotherapy is often impaired by the narrow therapeutic index of the cytotoxic drugs. Liposomes have been used as carriers for drug targeting to tumors. The biggest drawback has been MPS uptake. In disorders involving the MPS, advantage can be taken from this property hopefully 
leading to more effective treatment. Liposomal encapsulation of anti-cancer drugs changes the pharmacokinetics and, in the case of doxorubicin, reduces toxicity (10). Several different cytotoxic drugs have been successfully incorporated into liposomes $(11,12)$, and the reason for using teniposide was because of its major activity in the treatment of FHL and other hematologic malignancies $(2,5,6)$. Up to $10 \mathrm{~mol} \%$ teniposide has previously been incorporated into phosphatidylcholine liposomes $(13,14)$, but no in vivo data have been published.

To determine the maximum amount of teniposide possible to incorporate we used drug/lipid molar ratios of 1-15\%. During storage the teniposide formed crystals if the drug content was too high. After 2 mo only $50 \%$ of the drug was left in liposomal form if $3 \mathrm{~mol} \%$ teniposide was used. Drug loss from the liposomes containing $2 \%$ teniposide was negligable. Size stability of the preparations was good, and no aggregation occurred during storage in refrigerator $\left(8^{\circ} \mathrm{C}\right)$ for 3 mo.

After injection in mice the distribution of free teniposide to the extravascular binding sites was very rapid, and after 5 min only $10 \%$ of the injected dose remained in the plasma. The plasma clearance of liposome-encapsulated drug was slightly delayed and most pronounced with the largest liposomes (3 $\mu \mathrm{m})$. Clearance of the labeled phosphatidyl choline was slower, and this reflects tissue distribution of the lipid component of the liposomes.

By increasing the size of the liposomes the clearance rate by the MPS increased. This is well illustrated by the splenic uptake of teniposide $40 \mathrm{~min}$ post injection. When administered in 200-nm and 1- and 3- $\mu \mathrm{m}$ liposomes, the accumulation in the spleen was enhanced 2.6-, 6.8-, and 21-fold, compared with free teniposide. After 60 min the difference was even higher, and splenic levels after administration of teniposide in 3- $\mu \mathrm{m}$ liposomes was 39 times higher than after injection of free drug. Drug levels in the liver were high in all cases, inasmuch as it is a target organ for liposomes. Free teniposide is also accumulated in the liver and excreted in the bile (15). Distribution of radiolabeled phosphatidyl choline to liver and spleen was considerably higher, indicating a different distribution of the lipid component compared with teniposide. Lipids with a higher phase transition temperature such as disteaoryl phosphatidyl choline and a more multilamellar structure might have a better ability to protect the drug from leakage from the liposomes $(4,16)$. Previously we have tried to use cholesterol and disteaoryl phosphatidyl choline in teniposide liposomes, but these lipids impaired the incorporation of drug.

The discrepancy in our results between radioactive drug and radioactive phospholipid suggests the leakage of teniposide into the blood, alternatively the transfer of phospholipid from liposomes to plasma lipoproteins as previously described (17). In the present study the fate of the phospholipid differs from that of the drug. This illustrates how the choice of label affects the results when studying the effect of carriers on the in vivo distribution of drugs.

Bone marrow suppression is the dose limiting toxicity for teniposide. The extent of transcapillary transport of liposomes across the discontinuous capillaries in the bone marrow has not yet been determined (4). However, there is no transcapillary transport of liposomes larger than $200 \mathrm{~nm}$ in diameter through the discontinuous capillaries of the liver.

In a whole-body autoradiographic study the distribution of teniposide to the bone marrow was lower when it was administered in 3- $\mu \mathrm{m}$ liposomes compared with free teniposide (Vumon), suggesting less bone marrow toxicity (15).

In conclusion, the liposomal formulations change the pharmacokinetic behavior of teniposide. Particularly the distribution to the spleen is enhanced suggesting MPS uptake. Teniposide liposomes can possibly provide a more efficient therapy, with less toxic side effects, of certain histiocytic syndromes like FHL. Malignant histiocytosis, true histiocytic lymphomas, monocytic leukemias, and certain autoimmune disorders with a hyperactive phagocyte system such as idiopatic thrombocytopenia are other conditions that might benefit from treatment with teniposide liposomes.

Acknowledgments. The authors thank Dr. Michele Masquelier-Koitsalu for generous advice concerning the animal experiments, Birgitta Pettersson for skillful technical assistance, and Göran Karlsson for cryo-transmission electron microscopy measurements.

\section{REFERENCES}

1. Hwang K 1987 Liposome Pharmacokinetics. In: Ostro M (eds) Liposomes from Biophysics to Therapeutics. Marcel Dekker, New York, pp 109-156

2. Dombernowsky P, Hansen HH 1989 The epipodophyllotoxin derivatives VM 26 and VP 16: Experimental and clinical aspects. Eur J Haematol 42:49-57

3. Björkholm M 1990 Etoposide and Teniposide in the treatment of acute leukemia. Med Oncol Tumor Pharmacother 7:3-10

4. Ostro M, Cullis P 1989 Use of liposomes as injectable-drug delivery systems. Am J Hosp Pharm 46:1576-1587

5. Henter J, Elinder G 1991 Familial haemophagocytic lymphohistiocytosis. Clinical review based on the findings in seven children. Acta Paediatr Scand 80:269-277

6. Loy T, Diaz-Arias A, Perry M 1991 Familial erythrophagocytic lymphohistiocytosis Semin Oncol 18:34-39

7. Olson F, Hunt C, Szoka F, Vail W, Papahadjopoulos D 1979 Preparation of liposomes of defined size distribution by extrusion through polycarbonate membranes. Biochim Biophys Acta 557:9-23

8. Edwards K, Gustafsson J, Almgren M, Karlsson G 1993 Solubilization of lecithin vesicles by a cationic surfactant: Intermediate structures in the vesicle-micelle transition observed by cryo-transmission electron microscopy. J Colloid Interface Sci 161:299-309

9. Masquelier M, Vitols S, Peterson C 1986 Low-density lipoprotein as a carrier of antitumoral drugs: In vivo fate of drug-human low-density lipoprotein complexes in mice. Cancer Res 46:3842-3847

10. Sugarman SM, Perez-Soler R 1992 Liposomes in the treatment of malignancy: a clinical perspective. Crit Rev Oncol/Hematol 12:231-242

11. Juliano RL, Stamp D 1978 Pharmacokinetics of liposome-encapsulated anti-tumor drugs. Studies with vinblastine, actinomycin D, cytosine arabinoside and daunomycin. Biochem Pharmacol 27:21-27

12. Perez-Soler R 1989 Liposomes as carriers of antitumor agents: toward a clinical reality. Cancer Treat Rev 16:67-82

13. Wright SE, White JC 1986 Teniposide-induced changes in the physical properties of phosphatidylcholine liposomes; a calorimetric study. Biochem Pharmacol 35:27312735

14. Wright SE, White JC 1986 Membrane ordering effects of the anticancer agent VM-26. Biochim Biophys Acta 863:297-304

15. Liliemark E, Sjöström B, Liliemark J, Peterson C, Kållberg N, Larsson B Targeting of teniposide to the mononuclear phagocytic system (MPS) by incorporation in liposomes and submicron lipid particles; an autoradiographic study in mice. Leuk Lymphoma (in press)

16. Senior J, Gregoriadis G 1984 Methodology in assessing liposomal stability in the presence of blood, clearance from the circulation of injected animals and uptake by tissues. In: Gregoriadis G (ed) Liposome Technology. CRC Press, Boca Raton, FL, pp 263-294

17. Tall A 1980 Studies on the transfer of phosphatidylcholine from unilamellar vesicles into plasma high density lipoproteins in the rat. J Lipid Res 21:354--363 